\title{
Incorporating Thermal Fluctuations into the Immersed Boundary Method
}

\author{
P. R. Kramer ${ }^{\text {, }}$ C. S. Peskin ${ }^{\mathrm{b}}$ \\ ${ }^{a}$ Rensselaer Polytechnic Institute, \\ Department of Mathematical Sciences, Troy, NY 12180, USA \\ ${ }^{\mathrm{b}}$ New York University, \\ Courant Institute of Mathematical Sciences, \\ New York, NY 10012, USA
}

\begin{abstract}
We present a new computational method for simulating microscopic processes in physiology which accounts for stochastic thermal fluctuations and a dynamically evolving fluid environment. This latter feature is a principal advantage of our method relative to Brownian or Stokesian particle dynamics methods. Our approach builds on the "Immersed Boundary Method" of Peskin, which simplifies the coupling between the fluid and the immersed particles and membranes so as to avoid complex boundary problems.

Keywords: Immersed boundary method; Stochastic hydrodynamics; Fluctuationdissipation theorem; Thermal fluctuations; Brownian motion; Microfluid simulation

\section{Introduction}

The Immersed Boundary (IB) Method was introduced by Peskin [1] for efficient simulation of systems of fluids and elastic structures which are typical in biological
\end{abstract}


systems. The method has found numerous successful applications, mostly involving macroscopic organs and organisms, but also including some smaller scale phenomena such as microorganism swimming and amoeboid locomotion (Peskin $[2$, and references therein]). Another natural realm for application of the method is in microscopic processes occuring on a subcellular level such as the dynamics of vesicle transport, the operation of molecular motors, and the fluctuations of DNA. In physical terms, we contemplate systems with sizes extending from tens of nanometers to microns which are too large or too slow to be simulated in a satisfactory fashion through detailed molecular dynamics (Schlick [3]). Following the practice of conventional simulation approaches of such systems (Schlick [3], de la Torre and Bloomfield [4]), we model the physiological structures in terms of a collection of discrete particles with certain phenomenological force law interactions. We also treat the fluid as a continuous field. A major distinguishing feature of the Immersed Boundary (IB) approach is that it incorporates the dynamics of the fluid more faithfully via the Navier-Stokes equations, whereas most other methods (such as Brownian dynamics (Ermak and McCammon [5]) or Stokesian dynamics (Brady and Bossis [6], Sierou and Brady [7]) evolve the fluid according to a quasi-steady theory in which the fluid responds instantaneously to the forces applied to it by the immersed structures. One nice corollary of the IB method's explicit handling of the fluid is that topological constraints of the immersed structures (such as non-crossing of polymers) will be respected automatically.

Our main focus will be on the adaptation of the IB method to include thermal fluctuations which play a significant role on the dynamics of physiological structures on scales of microns and smaller. We present our approach in Section 2, and demonstrate in Section 3 that our simulation scheme appropriately reproduces 
several fundamental physical features of the thermally fluctuating system.

\section{Numerical Simulation Scheme}

In the Immersed Boundary (IB) method (Peskin [2]), the entire system of the fluid with immersed structures is treated as a constant density fluid obeying the NavierStokes equations, with the force term reflecting the elastic properties of the membrane and the interaction forces between particles and membranes. The locations at which the forces are applied depends on the positions of the particles and membranes, and these are updated by advection at a locally interpolated fluid velocity.

We assume the fluid domain $\Omega$ is a three-dimensional cube of side length $\ell$ with periodic boundary conditions. The velocity and pressure of the fluid are discretized on a common uniform mesh with grid spacing $h$, and their collective values at time step $n$ will be denoted $\mathbf{u}^{n}$ and $p^{n}$, respectively. We use the mathematical notation $\mathbb{Z}_{K}^{3}$ to denote the domain of grid points, indexed by integers modulo $K=\ell / h$, the number of grid points in each dimension.

The collection of immersed structures will be modeled as a finite collection of Lagrangian particles, located at positions $\mathbf{X}^{n}=\left\{\mathbf{X}_{\boldsymbol{\alpha}}^{n-1 / 2}\right\}_{\boldsymbol{\alpha} \in \mathcal{A}}$ immediately prior to time step $n$, where $\boldsymbol{\alpha}$ is a Lagrangian labelling index taking values from some finite set $\mathcal{A}$. The various stresses exerted by the immersed structures in response to deformations will be modelled in general through gradients of some interparticle potential $\tilde{\Phi}(\mathbf{X}, t)$.

In numerical simulations, the fluid and structure variables are updated alternately at each time step. To advance from time step $n$ to $n+1$, the Lagrangian 
particle positions evolve by advection through a locally interpolated fluid velocity:

$$
\mathbf{X}_{\alpha}^{n+1 / 2}=\mathbf{X}_{\alpha}^{n-1 / 2}+\Delta t \sum_{\mathbf{x} \in h \mathbb{Z}_{K}^{3}} \mathbf{u}^{n}(\mathbf{x}) \delta_{a}\left(\mathbf{X}_{\alpha}^{n-1 / 2}-\mathbf{x}\right) h^{3}
$$

The function $\delta_{a}(\mathbf{x})$ is a smoothed delta function with compact support on the cube $|\mathbf{x}| \leq 2 a$, satisfying $\int_{[-2 a, 2 a]^{3}} \delta_{a}(\mathbf{x}) d \mathbf{x}=1$ and other good discrete interpolation properties as discussed in Peskin [2]. Roughly speaking, it represents a local averaging of the fluid values over a region of length scale $a$ representing a geometric radius of the Lagrangian particle. The size parameter $a$ can be chosen as any integer multiple of the mesh spacing $h$. Depending on the application, the value of $a$ might remain fixed under refinements of the mesh $h$ if the physical effective particle size is already resolved.

The fluid variables are updated through the following semi-implicit version of the incompressible Navier-Stokes equations:

$$
\begin{aligned}
\rho\left(\frac{\mathbf{u}^{n+1}-\mathbf{u}^{n}}{\Delta t}+\mathcal{B}_{\mathbf{k}}^{(d)}\left(\mathbf{u}^{n}, \mathbf{u}^{n}\right)\right) & =-\nabla_{h} p^{n+1}+\mu L_{h}^{0} \mathbf{u}^{n+1}+\mathbf{f}_{\mathrm{d}}^{n}+\mathbf{f}_{\mathrm{T}}^{n}, \\
\boldsymbol{\nabla}_{h} \bullet \mathbf{u}^{n+1} & =0
\end{aligned}
$$

where $\Delta t$ is the time step, $\rho$ is the (constant) fluid density, $\mu$ is the dynamic viscosity, and $\mathbf{f}_{\mathrm{d}}^{n}$ is the systematic force density:

$$
\mathbf{f}_{\mathrm{d}}^{n}(\mathbf{x})=-\sum_{\boldsymbol{\alpha} \in \mathcal{A}} \nabla_{\mathbf{X}_{\boldsymbol{\alpha}}} \tilde{\Phi}\left(\left\{\mathbf{X}_{\boldsymbol{\alpha}}^{n+1 / 2}\right\}_{\boldsymbol{\alpha} \in \mathcal{A}}\right) \delta_{h}\left(\mathbf{x}-\mathbf{X}_{\boldsymbol{\alpha}}^{n+1 / 2}\right)
$$

and $\mathbf{f}_{\mathrm{T}}^{n}$ is the thermal force density which we describe in more detail below.

Following the usual practice (Peskin [2]), we take a centered difference approximation for the spatial derivatives $\nabla_{h}$ and $L_{h}^{0}$, and use a skew-symmetric central 
differencing scheme for the nonlinear operator:

$$
\mathcal{B}_{\mathbf{k}}^{(d)}\left(\mathbf{u}^{n}, \mathbf{u}^{n}\right) \equiv \frac{1}{2}\left(\mathbf{u}^{n} \cdot \nabla_{h} \mathbf{u}^{n}+\nabla_{h} \cdot\left(\mathbf{u}^{n} \otimes \mathbf{u}^{n}\right)\right)
$$

Since microscopic applications generally take place at low Reynolds number, the nonlinear term may even be meaningfully omitted. We insist on retaining the time derivative, however, since the thermal force and possibly some parts of the deterministic force fluctuate on a rapid time scale.

The thermal forcing is represented by the term

$$
\begin{aligned}
\mathbf{f}_{\mathrm{T}}^{n}(\mathbf{x}) & =\sum_{\mathbf{k} \in S_{K}} e^{2 \pi i \mathbf{k} \bullet \mathbf{x} / \ell} \mathbf{f}_{T, \mathbf{k}}^{n}, \\
\mathbf{f}_{T, \mathbf{k}}^{n} & =\sqrt{\frac{2 \rho k_{B} T \Gamma_{\mathbf{k}}}{\ell^{3} \Delta t}} \psi\left(\Gamma_{\mathbf{k}} \Delta t\right) \tilde{\mathbf{Z}}_{\mathbf{k}}^{n}
\end{aligned}
$$

where $T$ is the absolute temperature, $k_{B}=1.38 \times 10^{-23} \mathrm{~J} / \mathrm{K}$ is the Boltzmann constant,

$$
\Gamma_{\mathbf{k}} \equiv \frac{4 \mu}{\rho h^{2}} \sum_{j=1}^{3} \sin ^{2} \frac{\pi k_{j}}{K}
$$

is the dissipation rate of wavenumber $\mathbf{k}$ induced by the numerical viscosity operator $\mu \Delta_{h}$

$$
S_{K}=\mathbb{Z}_{K}^{3} \backslash\{(K, K, K)\}
$$

and the $\left\{\tilde{\mathbf{Z}}_{\mathbf{k}}^{n}\right\}_{\mathbf{k} \in S_{K}, n \in \mathbb{N}}$ are standard complex Gaussian random variables which are 
independent (other than reality condition $\tilde{\mathbf{Z}}_{-\mathbf{k}}^{n}=\left(\tilde{\mathbf{Z}}_{\mathbf{k}}^{n}\right)^{*}$ ) and which satisfy:

$$
\begin{aligned}
\left\langle\tilde{\mathbf{Z}}_{\mathbf{k}}^{n}\right\rangle & =0, \\
\left\langle\tilde{\mathbf{Z}}_{\mathbf{k}}^{n} \otimes \tilde{\mathbf{Z}}_{\mathbf{k}}^{n}\right\rangle & =0, \\
\left\langle\tilde{\mathbf{Z}}_{\mathbf{k}}^{n} \otimes\left(\tilde{\mathbf{Z}}_{\mathbf{k}}^{n}\right)^{*}\right\rangle & =1 .
\end{aligned}
$$

With $\psi \equiv 1$, the representation (2) for the thermal forcing is a straightforward temporal discretization of a white noise forcing specified by the fluctuationdissipation theorem (Kubo et al. [8]). If the nonlinear advection term is small, however, the introduction of the factor

$$
\psi(s)=\sqrt{\frac{(2+s)\left(e^{-s}+s-1\right)}{s^{2}}},
$$

permits the accurate simulation of the value of each system variable averaged over the time step, even if the time step is large compared to some (or even all) of the correlation times $1 / \Gamma_{\mathbf{k}}$ of the velocity modes. The time step must still resolve the dynamics of the immersed structures.

\section{Physicality of Thermal Forcing}

We had first investigated the possibility of applying the random forces directly on the Lagrangian particles, but we could not find a way to do this in a physically consistent manner within the IB framework (Kramer and Peskin [9]). We therefore elected instead to put the thermal forcing on the fluid variables, which will indirectly induce random motion of the immersed structures through the advection (1) by the thermally fluctuating velocity field. The fluctuation-dissipation theorem (Kubo et al. 
[8]) ensures that the fluid modes and vibrational modes of the immersed structures exhibit the correct statistics, even for typical non-equilibrium biological conditions (Kramer and Peskin [9], Español [10]). We must examine separately the question of whether the translational and rotational degrees of freedom of the immersed structures undergo a random Brownian motion of the appropriate amplitude. Here we focus on translational Brownian motion.

\subsection{Diffusion of Single Lagrangian Particle}

The fundamental measure of Brownian motion is the diffusion coefficient, which can be defined:

$$
D \equiv \lim _{t \rightarrow \infty} \frac{|\mathbf{X}(t)-\mathbf{X}(0)|^{2}}{6 t} .
$$

In practice, the long time limit is evaluated at a large but finite time. At low Reynolds number, the particle diffusivity in a physical system obeys the Einstein relation (Batchelor [11]):

$$
D=\frac{k_{B} T}{C_{D} \mu \sigma}
$$

where $\sigma$ is an effective particle radius and $C_{D}$ is a shape-dependent dimensionless drag coefficient $\left(C_{D}=6 \pi \approx 19\right.$ for a sphere). An asymptotic analysis shows that a force-free elementary Lagrangian particle with size parameter $a$ does undergo a diffusive motion over sufficiently long times, and its diffusivity has the correct scaling:

$$
D \approx \frac{k_{B} T}{C_{D}^{(I B)} \mu a}
$$

provided $h \ll a \ll \ell$, where the numerical constant $\tilde{C}_{D}^{(I B)} \approx 25$ (Kramer and Peskin [9], Kramer and Majda [12, 13]). Finite domain size and finite resolution corrections 
to this relationship are tabulated in Table 1. If one wishes to simulate, for example, the Brownian motion of a small sphere of radius $\sigma$ in terms of a single elementary Lagrangian particle, then the size parameter should be chosen as $a=6 \pi \sigma / \tilde{C}_{D}^{(I B)}$. An example trajectory of a free Brownian particle simulated by the IB Method is presented in Figure 1.

[Table 1 about here.]

[Figure 1 about here.]

\subsection{Correlated Diffusion between Particle Pairs}

The Brownian motion of different particles at low Reynolds number are coupled to each other. Indeed, consider an isolated pair of free particles at low Reynolds number with positions $\mathbf{X}_{1}(t)$ and $\mathbf{X}_{2}(t)$, situated at some time $t_{0}$ at $\mathbf{X}_{1}\left(t=t_{0}\right)=\mathbf{x}_{1}$ and $\mathbf{X}_{2}\left(t=t_{0}\right)=\mathbf{x}_{2}$. Then their subsequent random motion is correlated in the following way:

$$
\left\langle\left(\mathbf{X}_{1}(t)-\mathbf{x}_{1}\right) \otimes\left(\mathbf{X}_{2}(t)-\mathbf{x}_{2}\right)\right\rangle \approx 2 \mathrm{R}\left(\mathbf{x}_{2}-\mathbf{x}_{1}\right) t
$$

provided the time scale $t$ is much longer than the particle momentum relaxation time but shorter than the time it takes for the particle distance to change substantially. The spatial correlation structure obeys a slow power-law decay to zero:

$$
\mathrm{R}(\mathbf{r}) \approx \frac{k_{B} T}{8 \pi \mu|\mathbf{r}|}(\mathcal{I}+\hat{\mathbf{r}} \otimes \hat{\mathbf{r}}) \text { for }|\mathbf{r}| \gg \sigma
$$

where $\hat{\mathbf{r}}=\mathbf{r} /|\mathbf{r}|$. This asymptotic law can be shown to hold true for the IB method through asymptotic analysis (Kramer and Peskin [9], Kramer and Majda [12, 13]); 
an example is illustrated in Figure 2. The statistical behavior of the Lagrangian particles in the IB method suffers discrepancies from the correct physics at small separation distances (Kramer and Peskin [9], Kramer and Majda [12, 13]). The Brownian/Stokesian dynamics methods require a separate computational approach to handle nearby particles (Brady and Bossis [6], Sierou and Brady [7]), and the IB method might also be modified in some way to handle close particle encounters more precisely.

[Figure 2 about here.]

\section{Conclusion}

The Immersed Boundary method has been shown to be capable of simulating microscopic systems of a fluid with immersed structures in a way which includes many fundamental features of thermal fluctuations in a physically consistent manner.

\section{Acknowledgements}

The authors would like to thank Daniel Beard, George Oster, Eric Vanden-Eijnden, and Chris Wiggins for helpful discussions. CSP was supported in part by research grant R01 GM59875-01A1 from the National Institutes of Health. PRK was supported by a National Science Foundation postdoctoral fellowship.

\section{References}

[1] Peskin CS. Flow patterns around heart valves: a digital computer method for solving the equations of motion. Ph.D. thesis, Albert Einstein College of 
Medicine, 1972. University Microfilms no. 72-30, 378. 211 pp.

[2] Peskin CS. The immersed boundary method. Acta Numerica 2002; 11:479-517.

[3] Schlick T. Molecular Modeling and Simulation: An Interdisciplinary Guide, volume 21 of Interdisciplinary Applied Mathematics. Berlin: Springer-Verlag, 2002.

[4] de la Torre JG, Bloomfield VA. Hydrodynamic properties of macromolecular complexes. I. Translation. Biopolymers 1977; 16:1747-1764.

[5] Ermak DL, McCammon JA. Brownian dynamics with hydrodynamic interactions. J. Chem. Phys. 1978; 69(4):1352-1360.

[6] Brady JF, Bossis G. Stokesian dynamics. In: Annual review of fluid mechanics, volume 20 of Annu. Rev. Fluid Mech. Palo Alto, CA: Annual Reviews, 1988; $111-157$.

[7] Sierou A, Brady JF. Accelerated Stokesian Dynamics simulations. J. Fluid Mech. 2001; 448:115-146.

[8] Kubo R, Toda M, Hashitsume N. Statistical physics. II. Berlin: Springer-Verlag, second edition, 1991. Nonequilibrium statistical mechanics.

[9] Kramer PR, Peskin CS. An extension of the immersed boundary method including thermal fluctuations, 2003. In preparation.

[10] Español P. Stochastic differential equations for non-linear hydrodynamics. Physica A 1998; 248(1-2):77-96. 
[11] Batchelor GK. Developments in microhydrodynamics. In: Koiter WT, editor, Theoretical and Applied Mechanics. IUTAM Congress, Amsterdam, New York, Oxford: North Holland-Elsevier Science Publishers, 1976; 33-55.

[12] Kramer PR, Majda AJ. Stochastic mode reduction for particle-based simulation methods for complex microfluid systems, 2003. Submitted to SIAM J. Appl. Math.

[13] Kramer PR, Majda AJ. Stochastic mode reduction for the immersed boundary method, 2003. Submitted to SIAM J. Appl. Math. 


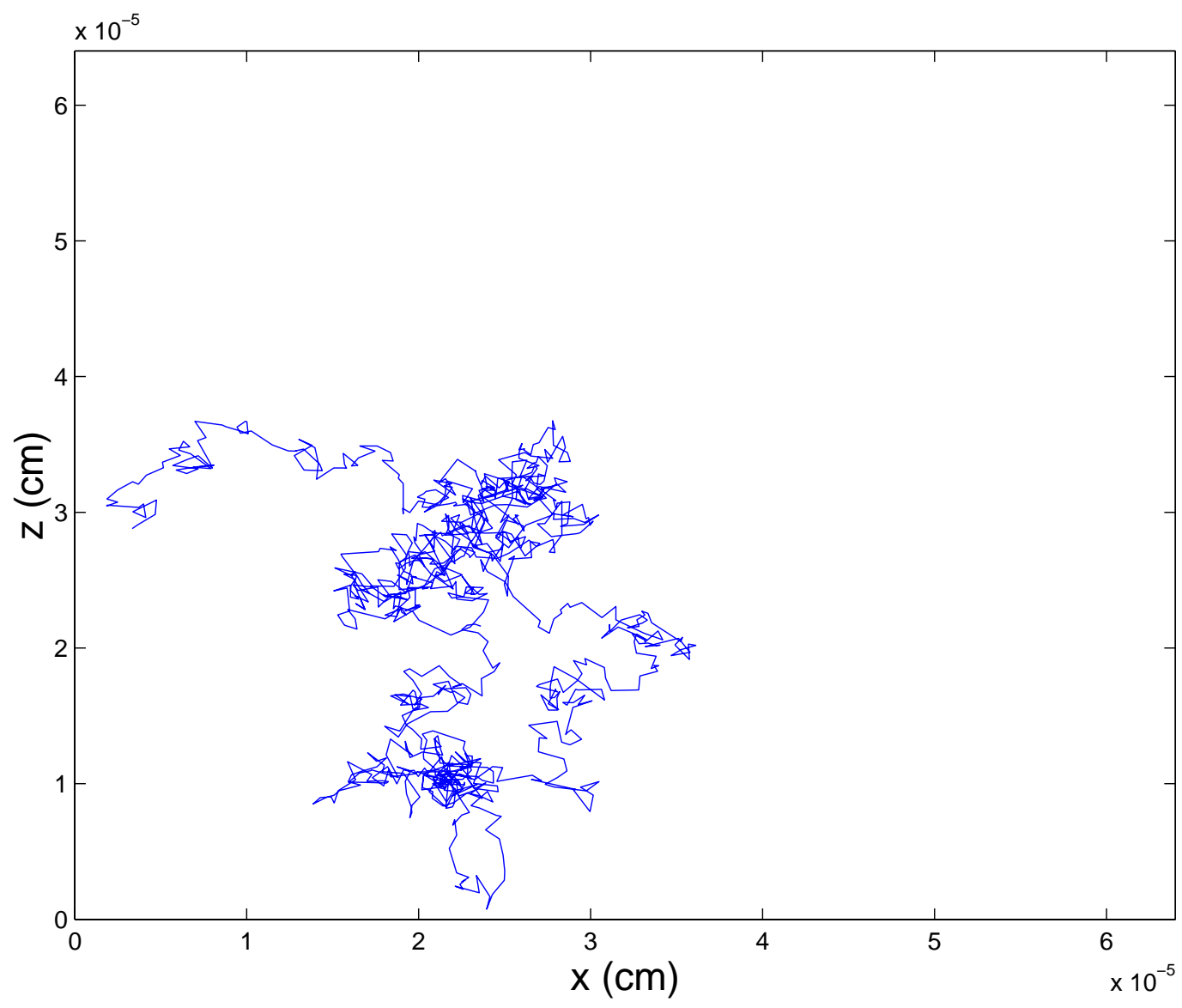

Figure 1: Two-dimensional projection of trajectory of three-dimensional Brownian motion of force-free particle simulated by Immersed Boundary Method. Particle size $a=10^{-6} \mathrm{~cm}$, grid size $h=10^{-6} \mathrm{~cm}$, time step $2 \times 10^{-7} \mathrm{~s}$, fluid density $\rho=1 \mathrm{~g} / \mathrm{cm}^{3}$, fluid viscosity $\mu=0.01 \mathrm{~g} /(\mathrm{cm} \mathrm{s})$ 

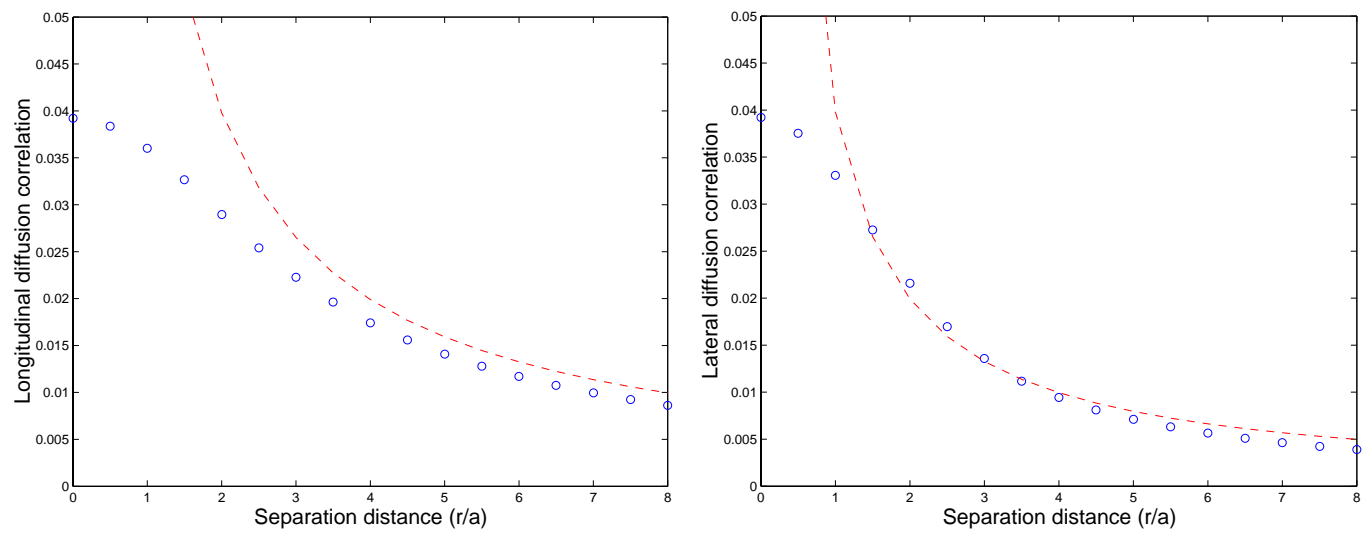

Figure 2: Left panel: Longitudinal diffusion correlation $\hat{\mathbf{r}} \cdot R(\mathbf{r}) \cdot \hat{\mathbf{r}}$. Right panel: Lateral diffusion correlation $\hat{\mathbf{n}} \cdot R(\mathbf{r}) \cdot \hat{\mathbf{n}}$ where $\hat{\mathbf{n}}$ is a unit vector perpendicular to $\hat{\mathbf{r}}$. The circles ('o') denote the calculated values for $\ell / a=128$ in the continuum limit $(a / h \rightarrow \infty)$ neglecting the nonlinear advection term, while the dashed line denotes the asymptotic law (6). 


\begin{tabular}{rrrr} 
& \multicolumn{3}{c}{$a / h$} \\
\cline { 2 - 4 }$\ell / a$ & 1 & 2 & $\infty$ \\
\hline 16 & 30.2 & 31.6 & 32.2 \\
32 & 26.6 & 27.6 & 28.0 \\
64 & 25.0 & 26.0 & 26.3 \\
128 & 24.3 & 25.2 & 25.5 \\
\hline \hline
\end{tabular}

Table 1: Finite domain size and finite resolution corrections to the scaling law (4) for the IB Method, neglecting nonlinear advection. The values in the table are $\tilde{C}_{D}^{(I B)} \equiv\left(k_{B} T\right) /(\mu a D)$, where $a$ is the effective particle size, $h$ is the grid spacing of the fluid lattice, and $\ell$ is the period length of the domain. 